Compared to what: What can we say about nonapeptide function and social behavior without a frame of reference?

Aubrey M. Kelly and Alexander G. Ophir*

Department of Psychology, Cornell University, Ithaca, NY 14853

\title{
*Corres pondence:
}

Alexander G. Ophir

224 Uris Hall

Department of Psychology

Cornell University

Ithaca, NY

14853, USA

Tel: (607) 255-3714

FAX: (607) 255-8433

E-mail: ophir@cornell.edu 


\begin{abstract}
Our understanding of behavior and mechanis $m$ is undermined by the absence of a frame of reference because relationships between individuals and species are without context. We highlight a need to be more comparative, using nonapeptide (vasopress in and oxytocin) modulation of social behavior as an example. We reconsider the use of model organisms and the term 'social' in this context, contrasting two popular models for nonapeptide regulation of social behavior. We then propose that a frame of reference should be established by studying mechanisms of behavior across taxa along the same continua. If we are to ever establish a unifying theory of behavior, we must transcend individual examples and determine the relative relationships of behavior and mechanism among and between species.
\end{abstract}

\author{
Keywords: \\ bed nucleus of the stria terminalis (BST) - lateral septum (LS) circuit \\ comparative approach \\ prairie vole (Microtus ochrogaster) \\ sociality \\ zebra finches (Taeniopygia guttata)
}




\section{Introduction}

Human interest in animal behavior predates recorded history, and depictions of animals in elaborate cave paintings made at least 35,000 years ago $[1,2]$ serve as a testament to mankind's fascination with animals. For centuries, humans gained an understanding of animal behavior by observing organis ms in their natural environment. However, it was not until the mid $20^{\text {th }}$ century that the study of animal behavior, known as ethology, emerged as a recognized scientific discipline. The founders of the field, most notably Tinbergen, Lorenz, and von Frisch, diverged from the behaviorist traditions in psychology by utilizing methods of observation of many different species. Their focus was on studying animals in their natural or a naturalistic environment, which deviated from the traditional approach of studying behavior strictly in a laboratory setting [3].

Advances in modern technology have increasingly channeled studies that focus on understanding behavioral mechanisms, particularly in neuroscience, toward the laboratory setting. This is largely due to the challenges that conducting mechanistic work in the field presents. Coinciding with the transition to studying behavior in the laboratory was the emergence of standard lab-bred animals, such as rats and mice [4]. Despite the convenience that lab animals provide, such species are often far removed from ancestral species that exist in the wild [5], which calls into question the ethological validity of findings. This can become problematic when attempting to translate findings from one species to another, as Beach [6] famously discussed. In addition, the consistent use of only a few standard species also puts us in danger of attempting to answer questions using animals that may not be suitable for the questions being asked. Research using standard lab animals is undoubtedly valuable; however, examining ethologically-relevant behavior in a variety of species will illuminate how behavioral mechanisms have evolved, and in turn can provide a deeper and more comprehensive understanding of behavior, which inherently has translatable value. It is also important that we ask appropriate questions in the species we study. To do so we need to consider the behavioral ecology of our study species and take precautions to carefully define the behaviors we examine.

In this review, we reconsider the use of model organisms and advocate for a need to be more comparative. To narrow the scope and allow for succinct and pertinent examples, we focus on nonapeptide modulation of social behavior because nonapeptides (vasopressin (VP) and oxytocin (OT)) are important generators of social behavioral diversity [7]. We begin by discussing the broad application of the term sociality and how it is defined. Next we present a case study that calls into question the common approaches used to investigate and understand social behavior. We then take a step back to consider 
the current state of our field and discuss the crucial need for taking a comparative approach when studying behavior.

\section{Defining sociality}

What is sociality? Although, historically, sociality was prominently used in reference to group living [8], in recent years, sociality has taken a rather broad meaning and serves as an umbrella term for numerous different types of social behavior that are in some way affiliative in nature [9-11•.]. Specific behaviors that fall under this umbrella include: groupsize preference, pair bonding, parental care, alloparental care, and side-by-side affiliative contact or huddling. At first glance, broadly referring to these individual behaviors as sociality does not seem overly problematic. However, there are often distinct mechanisms that modulate different types of social behavior. In zebra finches, for example, extended amygdalar VP promotes grouping but not pair bonding [12]. In this case, if sociality refers to both grouping and pair bonding, it would be inaccurate to say that extended amygdalar VP promotes sociality. In order to achieve a mechanistic understanding of behavior, we need to be careful with the language we select.

The term 'highly social' is similarly used loosely, often with respect to translational social neuroscience. What qualifies an animal as highly social? For instance, is it fair to categorize a species like the prairie vole as being highly social when individuals engage in pronounced affiliative social acts toward a pair bonded partner and offspring, yet also are profoundly territorial and exhibit high levels of aggression toward non-kin [13]? Inappropriate use of such a term within and between species risks a breakdown of the ability to generalize such a term, and may undermine the significance of describing a species or individual as 'highly social.' If such labels are too broadly applied, we risk losing meaning and may fall victim to making unfounded conclusions. For a comprehensive review on deconstructing sociality, see [11••]. It is extremely important that terms such as 'sociality' and 'highly social' not be used as general blanket terms, but rather are operationally defined and used with thoughtful precision.

\section{A tale of two species: When predictors of sociality do not generalize}

Virtually all tetrapods exhibit VP neurons in the medial extended amygdala, which lie primarily within the medial bed nucleus of the stria terminalis (BSTm) [14, 15]. This cell group is involved in numerous types of social behavior, and has been most thoroughly studied in relation to aspects of sociality in birds and rodents, and in particular zebra finches and prairie voles [16]. 
Zebra finches are biparental, monogamous, and highly gregarious (they prefer to affiliate in flocks of $\sim \mathbf{1 0 0}$ conspecifics). There is little doubt that this is a highly social species [17-19]. Comparative studies using a variety of monogamous finch species that differ selectively in their species-typical group size, including zebra finches, show that the BSTm VP cell group is involved in processing positive social stimuli $[20,21]$ and definitively promotes affiliative and grouping behavior [15, 22•]. A hallmark of the socialasocial continuum among finches is that gregarious finch species have significantly more BSTm VP cells and more V1a-like VP binding sites in the lateral septum (LS; a primary projection target of BSTm VP neurons) than do the territorial species [20, 23] (Figure 1). Based on this work, greater VP-ergic action in the BSTLS circuit seems to be an indicator of sociality.

Prairie voles are biparental, monogamous, and live primarily in social pairs, but can also form larger philopatric groups [24, 25]. Prairie voles are commonly described as a highly social and affiliative species, and the deeper understanding of

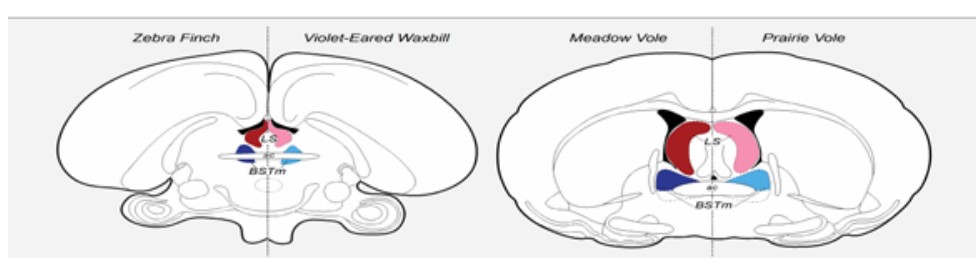

Figure 1. Relative expression profiles of VP in the BSTm (blue) and VlaR in the LS (red) for zebra finches (social/gregarious), violet-eared waxbills (asocial/ territorial), meadow voles (nonmonogamous), and prairie voles (monogamous) [see text for references]. Darker shades of red and blue indicate relatively higher density of either VP containing neurons or VlaR expression. Extensive work with estrildid finches indicates that high VP and VlaR in the BSTm-LS circuit facilitates social grouping, and questions the traditional characterization of prairie voles being 'highly social' in light of the expression patterns in voles presented in this figure, combined with behavioral traits such as species-specific patterns of aggression and social grouping [see 13]. mechanisms governing bonding and attachment between mating partners has propelled this species to be considered a model organism for human love, attachment, and social behavior [26•-28]. Interestingly, studies in finches and voles that examine the anatomy and function of the BSTm VP cell group in relation to sociality contradict one another. Prairie voles are often contrasted with the 'asocial,' non-monogamous meadow vole [29, 30]. Unlike the finch species comparisons, however, the prairie vole has fewer VP neurons in the BSTm and lower V1a-receptor densities in the LS than the purportedly asocial meadow vole [30, 31] (Figure 1).

Beyond the aforementioned anatomical differences, zebra finches and prairie voles exhibit functional differences in the BSTm-LS circuitry. Exposure to a same-sex conspecific elicits an increase in transcriptional (Fos) activity in BSTm VP neurons in both sexes of gregarious finch species [20]. We recently replicated this immediate early gene study in prairie voles by exposing males (sexually mature and socially-housed with samesex siblings) to a novel, same-sex conspecific for $90 \mathrm{~min}$ via interaction in a novel cage. 
While BSTm VP-Fos colocalization increases in male zebra finches in response to exposure to a novel conspecific male through a wire barrier or via a positive social interaction in a novel cage, interestingly, we observed no significant differences between the social-exposure group and the control group (empty cage) in VP-Fos colocalization in the BSTm of prairie voles (among other regions, including the anterior hypothalamus, paraventricular nucleus of the hypothalamus, and supraoptic nucleus of the hypothalamus; Mann Whitney U-test, all $p>0.4$; Kelly and Ophir, unpub data).

These contrasting findings between VP-ergic action in the BSTm-LS circuit and social grouping in finches and voles raise important questions. For example, are these differences a result of divergent evolution? In other words, have different neural mechanisms evolved to promote sociality in voles and finches that happen to express the opposite pattern in each species? While such an evolutionary outcome is plausible, it seems peculiar given the highly conserved nature of the social behavior network [32]. We would therefore expect it to be an uncommon occurrence for one mechanism to influence the same behavior in the opposite way. However, further comparative work will ultimately determine how common this might be. An alternative conclusion is that perhaps we have partially misinterpreted the behavioral ecology of these species groups, leading to a cavalier use of the term 'highly social.' For instance, although the prairie vole provides an outstanding opportunity to understand attachment, this species may be less appropriate to serve as a model for sociality, per se. This emphasizes the need to re-consider the meaning of 'social.'

\section{Box 1: Reconsidering model organisms}

The study of nonapeptide-mediated social behavior (along with many other areas of focus) falls subject to major differences in approach and perspective, often governed by different motivations. These differences often create distance and a lack of communication, resulting in a counterproductive divergence within the field. For example, if translational research is going to be truly effective, evolutionary perspectives must be incorporated.

Many of the early studies investigating the involvement of nonapeptides in social behavior were conducted in prairie voles (Microtus ochrogaster) [29, 33] - a species that is monogamous and biparental, and is now considered an emerging model organism for understanding the neurobiology of social behavior [28]. Interestingly, prairie voles stand apart from their classic rodent model counterparts, rats (Rattus norvegius) and mice (Mus musculus), because they have a well-documented behavioral ecology [24, 34, 35], and 
many labs use animals that are only a few generations removed from the wild, allowing for the desirable natural variation in social behaviors that is relevant to our own species [28]. This use of an outbred species is a notable and feasible tactic enabling maintenance and study of ethologically-relevant behaviors in the lab.

The lack of generalizability between the prairie vole and the zebra finch is somewhat alarming (see text), but perhaps this is mitigated by the fact that similarity between birds and mammals is expected to be reduced compared to the expected similarity within mammals. Because of prairie voles' current prominence in translational social neuroscience, it is crucial that results are generalizable if they are to provide meaningful insight into human heath or more general phenomena. Nevertheless, resting the majority of our knowledge on one species or clade can be risky. For example, even within mammals, aspects of affiliation are modulated differently between prairie voles and marmosets, such that OT receptor blockade in marmosets does not alter partner preference behavior [36, 37] as it does in voles. When results do not replicate across species, then doubt about the general function should be raised.

Where should comparison across taxa begin? Many good options to understand nonapeptide function and social behavior exist. Indeed, advances in understanding nonapeptide function and social behavior in the lab have been made in several examples of tractable, non-traditional species. These examples include meadow voles [38], mandarin voles [39], tuco tucos [40], deer mice [41, 42], African striped mice [43, 44], guinea pigs [45], tree shrews [46•], titi monkeys [47•], rhesus macaques $[48,49]$, zebra finches $[50,51]$, anole lizards [52], tree frogs [53], butterfly fish [54], and cichlid fishes [55, 56].

Although model organisms are us eful for furthering scientific discovery, translational insights into behavioral dimensions that are evolutionarily labile will be most readily obtained through identification of common mechanisms across several independent cases of behavioral evolution. Unfortunately, it is often unpractical to bridge these gaps when labs work in isolation, and thus, collaboration or working toward a collective goal is necessary. Integrating knowledge and perspectives across research priorities and motivations will begin to reduce the divide, while advancing the common goal: to understand behavior.

Moving forward: Establishing a unified framework through comparison

The example above emphasizes the need to take a more broadly comparative approach to studying behavior. While finches and voles appear to be superficially similar 
for the purposes of studying social behavior, they exhibit nuanced and important differences in sociality. Perhaps not surprisingly, the nonapeptide mechanisms underlying sociality also differ between the two species. If we are to truly understand the evolution of social behavior, we must consider the fact that various aspects of social behavior evolved independently many times, and we cannot assume that relevant mechanisms have evolved similarly in all species.

In order to build a solid foundation on which to study behavior and the underlying mechanisms, we must consider several important factors. Principally, we must use comparative approaches to examine a diversity of species within and across taxa. Doing so will ultimately determine the fundamental principle components for particular social behaviors (Figure 2). On the other hand, and equally important, we must consider the behavioral ecology of an organism and how this shapes components of social behavior. This approach helps explain the significant species-specific subtleties that govern the same behaviors (Figure 2). A combination of these approaches will ultimately provide a picture of the primary evolutionary drivers for those behaviors, both in terms of identifying unifying principles that generalize across taxa, and the singularities that make animals different.

A necessary major step toward achieving a unified theory of behavior must take a more comparative approach. Without a fundamental baseline that captures the similarity across taxa (Figure 2), comparisons lack reference. For example, it seems clear that VP-ergic action in the BSTm-LS circuit of both voles and finches is important for modulating social grouping or antisocial territoriality (see above and 13), suggesting a clear link between this mechanism and behavior. However, in isolation, it is difficult to determine if prairie voles, zebra finches, or both, represent outliers, or if one or both represent a more common relationship in the social brain (Figure 2). Zebra finches live in extremely arid environments, and living in large groups serves a protective function [18]. Perhaps the extreme sociality seen in zebra finches is the result of evolution pushing this species away from the norm. Prairie voles demonstrate social monogamy, a mating system that is often associated with living in resource depleted and highly competitive ecological environments [57]. Perhaps evolution has pushed the mechanisms that regulate their social behavior away from a baseline in a different direction. 
While there are countless differences

in social behavior exhibited across species, there exist common threads within all animals that follow one basic evolutionary trajectory. In some cases, for example where sampling is limited, phylogenetic corrections may be necessary. However, their appropriateness will be based on the breadth of the sampling (e.g., the utility of such corrections dwindles as sampling broadens) and the feasibility of having a well-resolved multitaxonomic tree on which to base such comparisons [see 58-63]. Such issues not withstanding, it is imperative that we elucidate the central tendency that stems from the emergence of social behavior beginning billions of years ago, and that has persisted in all extant

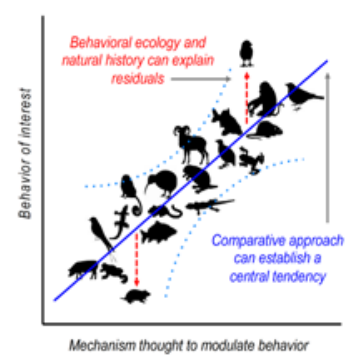

Figure 2. A hypothetical visualization of how study of a particular brain-behavior relationship across taxa can begin to reveal a central tendency for a 'phylogenetic norm' (blue line). We note that although we have presented this figure as linear, the best-fit curve could just as well be curvilinear or follow some other function, and the scale may be ordinal or logarithmic. Each silhouette represents a hypothetical place along two continua in which a given species may fall (light blue dotted lines represent hypothetical confidence intervals that capture the natural variation around the central tendency). Deviations (residuals; red dashed lines) from the central tendency represent the outcome of evolutionary pressures to depart from the norm, presumably at a cost that is outweighed by the benefit the deviation provides. Establishing such a baseline enables greater power in determining what is normal and abnormal, and places what we know in

social animals. Indeed, social behavior has evolved independently numerous times.

Studying a small sample of species will fail to capture the common tendencies present since the dawn of social behavior and seen throughout evolutionary history. In order to elucidate such unifying principles, we need to "think more phylogenetically." From this perspective, each species is, in essence, an $n=1$. In order to obtain a greater understanding of the most basic principles that operate in all creatures, we need greater power - power achieved from thoroughly understanding social behavior systems of interest in multiple species across various taxa. It is naïve to assume that focusing most of our efforts on the study of social behavior in prairie voles, zebra finches, rhesus macaques, and cichlids is enough. Compiling a larger data set of many more species will establish a baseline that can serve as a frame of reference. This, in turn, will allow scientists to effectively answer the myriad of questions relating to social behavior, the evolution of this behavior, the mechanis ms that underlie this behavior, and how this behavior extends to other behavioral domains.

\section{Concluding remarks}


The topic of social behavior, and the nonapeptide influences therein, has received a great amount of attention, and this has clearly advanced both our basic understanding of social behavior and provided exciting avenues to improve human heath. However, it is clear that we have only scratched the surface. Moreover, a basic tenet of the scientific method is to create comparisons and to search for contrasts from a norm, or control. In order to interpret what we have learned from any given species, we must be able to know the extent to which what we have learned deviates from other cases. A point of comparison can only be achieved by compiling a rich understanding of behaviors and the mechanisms that drive them in an array of species. Pursuing this lofty goal promises to illuminate how and why behavioral mechanisms evolved.

It is extremely important to have an in-depth understanding of social behavior in one species (for example, nonapeptide systems may be most thoroughly understood in prairie voles). However, social behavior is, in and of itself, complex, even without considering the background on which a particular species lays (i.e., behavioral ecology, environmental influences, etc.). With more comparative research, we can reveal basic commonalities in nonapeptide modulation of social behavior that are present in all species. Doing so will provide a reference point to understand where organisms of interest fall relative to each other, and provide a taxonomic-wide view of what is 'normal.' Indeed, it is this central tendency that has created a background from which plasticity and variation emerges. Only after such a reference point has been established, can we then begin to investigate this residual 'noise' and examine the selective pressures (environment, context, etc.) responsible for driving evolutionary processes in a new direction. For those interested in translational research, understanding where and how social behavior has evolved across taxa can help elucidate the distinct forces that have shaped human social behavior, and provide deeper insight into human nature.

\section{Acknowledgements}

We thank Leah C. Wilson for assistance with histology and cell counts for the prairie vole IE G study. The authors acknowledge the support of the National Institutes of Health (Eunice Kennedy Shriver National Institute of Child Health and Human Development F32 HD081959 to AMK, R15 HD065604 to AGO, R01 HD079573 to AGO) and the National Science Foundation (IOS 1354760 to AGO). We would like to especially acknowledge J ames ( $\mathrm{im}$ ) L. Goodson (1965-2014) for his dedication to the pursuit of utilizing a comparative approach to studying the neurobiology of social behavior. He was a dear friend, colleague, and mentor and he is sincerely missed. 


\section{Figure Captions}

Figure 1. Relative expression profiles of VP in the BSTm (blue) and V1aR in the LS (red) for zebra finches (social/gregarious), violet-eared waxbills (asocial/territorial), meadow voles (non-monogamous), and prairie voles (monogamous) [see text for references]. Darker shades of red and blue indicate relatively higher density of either VP containing neurons or V1aR expression. Extensive work with estrildid finches indicates that high VP and V1aR in the BSTm-LS circuit facilitates social grouping, and questions the traditional characterization of prairie voles being 'highly social' in light of the expression patterns in voles presented in this figure, combined with behavioral traits such as species-specific patterns of aggression and social grouping [see 13].

Figure 2. A hypothetical visualization of how study of a particular brain-behavior relationship across taxa can begin to reveal a central tendency for a 'phylogenetic norm' (blue line). We note that although we have presented this figure as linear, the best-fit curve could just as well be curvilinear or follow some other function, and the scale may be ordinal or logarithmic. Each silhouette represents a hypothetical place along two continua in which a given species may fall (light blue dotted lines represent hypothetical confidence intervals that capture the natural variation around the central tendency). Deviations (residuals; red dashed lines) from the central tendency represent the outcome of evolutionary pressures to depart from the norm, presumably at a cost that is outweighed by the benefit the deviation provides. Establishing such a baseline enables greater power in determining what is normal and abnormal, and places what we know in an evolutionary context.

\section{References and Recommended Reading}

Papers of particular interest, published within the period of review, have been highlighted as:

- of special interest

-• outstanding interest

1. Aubert M, Brumm A, Ramli M, Sutikna T, Saptomo EW, Hakim B, Morwood MJ , van den Bergh GD, Kinsley L, Dosseto A: Pleistocene cave art from Sulawesi, Indonesia. Nature 2014, 514: 223-227. 
2. Valladas H, Clottes J , Geneste J M, Garcia MA, Arnold M, Cachier H, TisneratLaborde N: Palaeolithic paintings. Evolution of prehistoric cave art. Nature 2001, 413: 479.

3. Burkhardt RW: Patterns of Behavior: Konrad Lorenz, Niko Tinbergen, and the founding of ethology. Univ. of Chicago Press 2005.

4. Krinke GJ : The laboratory rat: Handbook of experimental animals. Academic Press 2000.

5. Castle WE: The Domestication of the Rat. Proc. Natl. Acad. Sci. U S A 1947, 33: 109-117.

6. Beach FA: The snark was a boojum. Am Psychol 1950, 5: 115-124.

7. Goodson J L: Nonapeptides and the evolutionary patterning of sociality. Prog. Brain Res. 2008, 170: 3-15.

8. Alexander RD: The evolution of social behavior. Ann. Rev. Ecol. Syst. 1974, 5: 325383.

9. Carter CS, Grippo AJ , Pournajafi-Nazarloo H, Ruscio MG, Porges SW: Oxytocin, vasopressin and sociality. Prog. Brain Res. 2008, 170: 331-336.

10. Donaldson ZR, Young LJ : Oxytocin, vasopressin, and the neurogenetics of sociality. Science 2008, 322: 900-904.

11. $\cdot$ Goodson J L: Deconstructing sociality, social evolution and relevant nonapeptide functions. Psychoneuroendocrinology 2013, 38: 465-478.

Goodson delves into the body of literature examining sociality and provides a thorough review on how the term sociality is broadly used in the research community. He discusses the challenges such a loose use of the term 'sociality' poses for studying nonapeptide-mediated social behavior, and presents comparative data from a variety of finch species to highlight the functional evolution of nonapeptide systems in relation to sociality.

12. Kelly AM, Goodson J L: Functional significance of a phylogenetically widespread sexual dimorphism in vasotocin/vasopressin production. Horm. Behav. 2013, 64: 840-846.

13. Ophir AG: Towards meeting Tinbergen's challenge. Horm. Behav. 2011, 60: 22-27.

14. De Vries GJ , Panzica GC: Sexual differentiation of central vasopressin and vasotocin systems in vertebrates: different mechanisms, similar endpoints. Neuroscience 2006, 138: 947-955.

15. Kelly AM, Kingsbury MA, Hoffbuhr K, Schrock SE, Waxman B, Kabelik D, Thompson RR, Goodson J L: Vasotocin neurons and septal $V_{1 a}$-like receptors potently modulate songbird flocking and responses to novelty. Horm. Behav. 2011, 60: 12-21. 
16. Kelly AM, Goodson J L: Social functions of individual vasopressin-oxytocin cell groups in vertebrates: What do we really know? Front. Neuroendocrinol. 2014.

17. Goodwin D: Estrildid Finches of the World, 1st Edition Edition, Ithaca, NY: Cornell Univ Press 1982.

18. Zann RA: The Zebra Finch: A Synthesis of Field and Laboratory Studies, Oxford University Press, USA 1996.

19. Goodson J L, Evans AK, Lindberg L, Allen CD: Neuro-evolutionary patterning of sociality. Proc. Biol. Sci. 2005, 272: 227-235.

20. Goodson J L, Wang Y: Valence-sensitive neurons exhibit divergent functional profiles in gregarious and asocial species. Proc. Natl. Acad. Sci. USA 2006, 103: 17013-17017.

21. Goodson J L, Rinaldi J , Kelly AM: Vasotocin neurons in the bed nucleus of the stria terminalis preferentially process social information and exhibit properties that dichotomize courting and non-courting phenotypes. Horm. Behav. 2009, 55: $197-$ 202.

22. - Kelly AM, Goodson J L: Behavioral relevance of species-specific vasotocin anatomy in gregarious finches. Front. Neurosci. 2013, 7: 242. Nonapeptide cell groups are highly conserved across taxa. This study examines the behavioral functions of the BSTm VP cell group in the modestly gregarious Angolan blue waxbill to provide a comparis on to similar studies conducted in zebra finches. While the BSTm VP neurons do not modulate group size preference as in zebra finches, these cells promote social contact in the waxbills. These results highlight species-specific effects of nonapeptides on sociality.

23. Goodson J L, Evans AK, Wang Y: Neuropeptide binding reflects convergent and divergent evolution in species-typical group sizes. Horm. Behav. 2006, 50: 223-236.

24. Getz LL, McGuire, B., Pizzuto, T., Hofmann, J .E., Frase, B.: Social organization of the prairie vole (Microtus ochrogaster). J. Mammal. 1993, 74: 44-58.

25. J acquot J J, Solmon NG: Experimental manipulation of territory occupancy: effects on immigration of female prairie voles. J. Mammal. 2004, 85: 1009-1014.

26. - Carter CS, Porges SW: The biochemistry of love: an oxytocin hypothesis. EMBO reports 2013, 14: 12-16.

Carter and Porges consider the topic of 'love' from a physiological persepctive. They explore the evolutionary pathways that have led to social behavior and the capacity for love. They then discuss the important contributions that oxytocin and vasopressin make toward facilitating behaviors important for the expression of love. Finally they explore oxytocin's ability to literally repair a broken heart (cardiac tissue repair). 
27. Insel TR: The challenge of translation in social neuroscience: a review of oxytocin, vasopressin, and affiliative behavior. Neuron 2010, 65: 768-779.

28. McGraw LA, Young LJ : The prairie vole: an emerging model organism for understanding the social brain. Trends Neurosci. 2010, 33: 103-109.

29. Insel TR, Wang ZX, Ferris CF: Patterns of brain vasopressin receptor distribution associated with social organization in microtine rodents. J. Neurosci. 1994, 14: 5381-5392.

30. Wang Z: Species differences in the vasopressin-immunoreactive pathways in the bed nucleus of the stria terminalis and medial amygdaloid nucleus in prairie voles (Microtus ochrogaster) and meadow voles (Microtus pennsylvanicus). Behav. Neurosci. 1995, 109: 305-311.

31. Bamshad M, Novak MA, De Vries GJ : Sex and species differences in the vasopress in innervation of sexually naive and parental prairie voles, Microtus ochrogaster and meadow voles, Microtus pennsylvanicus. J. Neuroendocrinol. 1993, 5: 247-255.

32. O'Connell LA, Hofmann HA: The vertebrate mesolimbic reward system and social behavior network: A comparative synthesis. J. Comp. Neurol. 2011, 519: 3599-3639.

33. Insel TR, Shapiro LE : Oxytocin receptor distribution reflects social organization in monogamous and polygamous voles. Proc. Natl. Acad. Sci. U S A 1992, 89: 59815985.

34. Tamarin RH, Ostfeld RS, Pugh SR, Bujalski G: Social systems and population cycles in voles. Birkhauser Basel: Advances in Life Sciences 1990.

35. Getz LL, Hofmann J E: Social organization in free-living prairie voles, Microtus ochrogaster. Behav. Ecol. Sociobiol. 1986, 18: 275-282.

36. Smith AS, Agmo A, Birnie AK, French J A: Manipulation of the oxytocin system alters social behavior and attraction in pair-bonding primates, Callithrix penicillata. Horm. Behav. 2010, 57: 255-262.

37. Young LJ : The neuroendocrinology of the social brain. Front. Neuroendocrinol. 2009, 30: 425-428.

38. Beery AK, Zucker I: Oxytocin and same-sex social behavior in female meadow voles. Neuroscience 2010, 169: 665-673.

39. Qiao X, Yan Y, Wu R, Tai F, Hao P, Cao Y, Wang J : Sociality and oxytocin and vasopressin in the brain of male and female dominant and subordinate mandarin voles. J. Comp. Physiol. A, 2014, 200: 149-159.

40. Beery AK, Lacey EA, Francis DD: Oxytocin and vasopressin receptor distributions in a solitary and a social species of tuco-tuco (Ctenomys haigi and Ctenomys sociabilis). J. Comp. Neurol. 2008, 507: 1847-1859. 
41. de J ong TR, Chauke M, Harris BN, Saltzman W: From here to paternity: neural correlates of the onset of paternal behavior in California mice (Peromyscus californicus). Horm. Behav. 2009, 56: 220-231.

42. Steinman MQ, Laredo SA, Lopez EM, Manning CE, Hao RC, Doig IE, Campi KL, Flowers AE, Knight J K, Trainor BC: Hypothalamic vasopressin systems are more sensitive to the long term effects of social defeat in males versus females. Psychoneuroendocrinology 2015, 51: 122-134.

43. Schradin C, Kenkel W, Krackow S, Carter CS: Staying put or leaving home: endocrine, neuroendocrine and behavioral consequences in male African striped mice. Horm. Behav. 2013, 63: 136-143.

44. Schradin C, Larke RH, Bales KL: Growing up in the family or growing up alone influences behavior and hormones, but not arginine vasopressin receptor la expression in male African striped mice. Physiol. Behav. 2014, 129: 205-213.

45. Wallner B, Dittami J , Machatschke I: Social stimuli cause changes of plasma oxytocin and behavior in guinea pigs. Biol. Res. 2006, 39: 251-258.

46. - Ni RJ , Shu YM, Wang J , Yin J C, Xu L, Zhou J N: Distribution of vasopressin, oxytocin and vasoactive intestinal polypeptide in the hypothalamus and extrahypothalamic regions of tree shrews. Neuroscience 2014, 265: 124-136. Tree shrews are the closest relative to primates and make for an excellent species for examining stress-related, social, and aggressive behaviors. Little is known about the distribution of neuropeptides in tree shrews. This study provides a detailed mapping of VP, OT, and vasoactive intestinal polypeptide anatomy, and reveals sex differences in addition to similarities and differences with peptide distribution in other mammals.

47. - Freeman SM, Walum H, Inoue K, S mith AL, Goodman MM, Bales KL, Young LJ : Neuroanatomical distribution of oxytocin and vasopressin la receptors in the socially monogamous coppery titi monkey (Callicebus cupreus). Neuroscience 2014, 273: 12-23.

Coppery titi monkeys are socially monogamous primates that are used in the field and lab to study parenting and pair bonding. Behavioral pharmacology has shown that VP and OT are involved in affiliative behaviors in this species; however the neural mechanisms by which these peptides modulate behavior remain unclear. This study provides insight into the neural circuits modulated by VP and OT that are known to affect species-specific social behavior. The study details the distribution of VP and OT receptors and investigates candidate antagonists for future pharmacological manipulation of these receptors. 
48. Weinstein TA, Bales KL, Maninger N, Hostetler CM, Capitanio J P: Early involvement in friendships predicts later plasma concentrations of oxytocin and vasopressin in juvenile rhesus macaques (Macaca mulatta). Front. Behav. Neurosci. 2014, 8: 295.

49. Modi ME, Connor-Stroud F, Landgraf R, Young LJ , Parr LA: Aerosolized oxytocin increases cerebrospinal fluid oxytocin in rhesus macaques. Psychoneuroendocrinology 2014, 45: 49-57.

50. Kelly AM, Goodson J L: Hypothalamic oxytocin and vasopressin neurons exert sexspecific effects on pair bonding, gregariousness and aggression in finches. Proc. Natl. Acad. Sci. USA 2014, 111: 6069-6074.

51. Lowrey EM, Tomaszycki ML: The formation and maintenance of social relationships increases nonapeptide mRNA in zebra finches of both sexes. Behav. Neurosci. 2014, 128: 61-70.

52. Kabelik D, Alix VC, Burford ER, Singh LJ : Aggression- and sex-induced neural activity across vasotocin populations in the brown anole. Horm. Behav. 2013, 63: 437-446.

53. Lutterschmidt DI, Wilczynski W: Sexually dimorphic effects of melatonin on brain arginine vasotocin immunoreactivity in green treefrogs (Hyla cinerea). Brain Behav. Evol. 2012, 80: 222-232.

54. Dewan AK, Tricas TC: Arginine vasotocin neuronal phenotypes and their relationship to aggressive behavior in the territorial monogamous multiband butterflyfish, Chaetodon multicinctus. Brain Res. 2011, 1401: 74-84.

55. Huffman LS, Hinz FI, Wojcik S, Aubin-Horth N, Hofmann HA: Arginine vasotocin regulates social ascent in the African cichlid fish Astatotilapia burtoni. Gen. Comp. Endocrinol. 2014.

56. Almeida O, Gozdowska M, Kulczykowska E, Oliveira RF: B rain levels of argininevasotocin and isotocin in dominant and subordinate males of a cichlid fish. Horm. Behav. 2012, 61: 212-217.

57. Emlen ST, Oring LW: Ecology, sexual selection, and the evolution of mating systems. Science 1977, 197: 215-223.

58. Weathers WW, Siegel RB: Body size establishes the scaling of avian postnatal metabolic rate: an inter- specific analysis using phylogenetically independent contrasts. Ibis 1995, 137: 532-542.

59. Westoby M, Leishman MR, Lord J M: On mis-interpreting the 'phylogenetic correction'. J. Ecol. 1995, 83: 531-534.

60. Ricklefs RE, Starck J M: Applications of phylogenetically independent contrasts: a mixed progress report. Oikos 1996, 77: 167-172. 
61. Bjorklund M: Are 'comparative methods' always necessary? Oikos 1997, 80: 607612.

62. Losos J B: Phylogenetic niche conservatism, phylogenetic signal and the relationship between phylogenetic relatedness and ecological similarity among species. Ecol. Lett. 2008, 11: 995-1007.

63. Gillilooly J F, Ophir AG: The energetic basis of acoustic communication. Proc. $R$. Soc. B. 2010, 277: 1325-1331. 


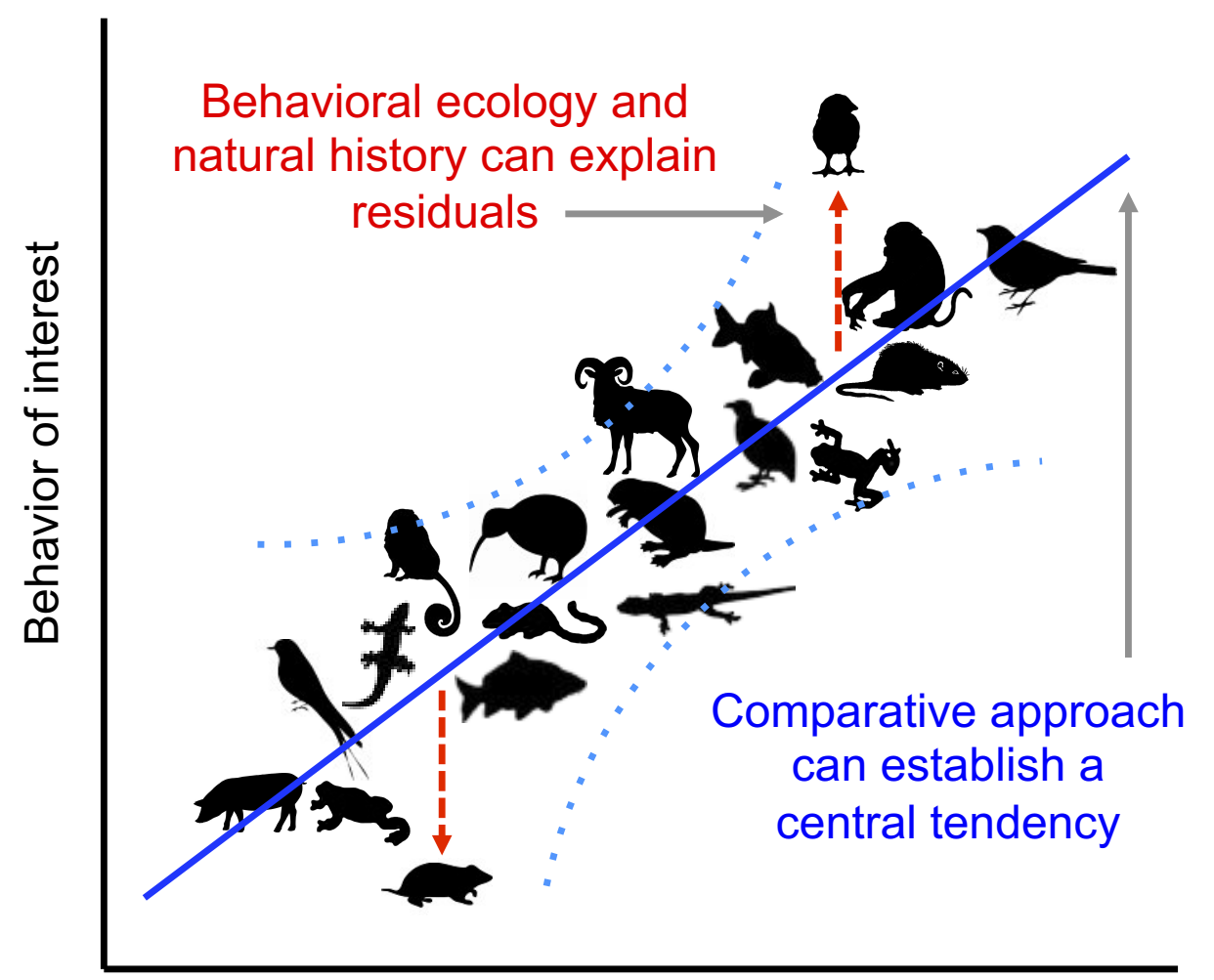

Mechanism thought to modulate behavior 